\title{
Forschungsliteratur und gedruckte Quellen
}

Eric Agrikoliansky, La gauche, le libéralisme politique et les droits de l'homme, in: Jean-Jacques Becker/Gilles Candar (Hrsg.), Histoire des gauches en France, Bd. 2, Paris 2005, S. 524-541.

Akten zur Auswärtigen Politik der Bundesrepublik Deutschland. Hrsg. im Auftrag des Auswärtigen Amts vom Institut für Zeitgeschichte.

Raymond Aron, Les articles de politique internationale dans Le Figaro de 1947 à 1977, Bd.3: Les Crises (Février 1965 à avril 1977), Paris 1997.

Raymond Aron, La Révolution introuvable. Réflexions sur les événements de mai, Paris 1968.

Philippe Artières/Laurent Quéro/Michelle Zancarini-Fournel (Hrsg.), Le groupe d'information sur les prisons. Archives d'une lutte, Paris 2003.

Amin Ash (Hrsg.), Post-Fordism. A Reader, Oxford/Cambridge 1994.

Robert Badinter (Hrsg.), Liberté, libertés. Réflexions du comité pour une charte des libertés, Paris 1976.

Ralf Balke, Israel, 3. neu bearb. Aufl., München 2007.

Arnulf Baring, Gustav Heinemann und der Machtwechsel, in: Regina Krane (Hrsg.), Nachdenken. Gustav Heinemann und seine Politik, Bonn 1999, S. 41-53.

Arnulf Baring, Machtwechsel. Die Ära Brandt-Scheel, Stuttgart 1982.

Alain Barjot (Hrsg.), La sécurité sociale, son histoire à travers les textes, Bd. 3: 1945-1981, Paris 1988.

Raymond Barre, Une politique pour l'avenir, Paris 1981.

Ulrich Beck, Risikogesellschaft. Auf dem Weg in eine andere Moderne, Frankfurt am Main 1986.

Jean-Jacques Becker, Crises et alternances 1974-1995, Paris 1998.

Jean-Jacques Becker, Crises et alternances 1974-2000, Paris 2002.

Daniel Bell, The Coming of Post-Industrial Society: A Venture in Social Forecasting, New York 1973, deutsche Ausgabe: Die nachindustrielle Gesellschaft, Reinbek bei Hamburg 1979.

Manfred Beller, Lenz in Arkadien: Peter Schneiders Italienbild von Süden betrachtet, in: Arcadia, Sonderheft: Horst Rüdiger zum 70. Geburtstag, Januar 1978, S. 91-105.

Alain Beltran, La question énergétique en France de 1960 à 1974: dépendance, crise et rôle de l'État, in: Éric Bussière (Hrsg.), Georges Pompidou face à la mutation économique de l'Occident 1969-1974, Paris 2003, S. 191-223.

Uwe Berlit/Horst Dreier, Die legislative Auseinandersetzung mit dem Terrorismus, in: Fritz Sack/Heinz Steinert (Hrsg.), Analysen zum Terrorismus, Bd. 4.2: Protest und Reaktion, Opladen 1984, S. 227-318.

Jacques Berne, La campagne présidentielle de Valéry Giscard d'Estaing en 1974, Paris 1981.

Patrick Bernhard, Zivildienst zwischen Reform und Revolte. Eine bundesdeutsche Institution im gesellschaftlichen Wandel 1961-1982, München 2005.

Serge Berstein/Jean-François Sirinelli (Hrsg.), Les années Giscard. Les réformes de société, Paris 2007.

Adolf M. Birke, Die Bundesrepublik Deutschland. Verfassung, Parlament und Parteien 19451998, 2. Aufl., ergänzt und aktualisiert von Udo Wengst, München 2010.

Ernst Bloch, Das Prinzip Hoffnung, Frankfurt am Main 1959.

François Bloch-Lainé, Pour une réforme de l'entreprise, Paris 1963.

Frank Bösch, Die Krise als Chance. Die Neuformierung der Christdemokraten in den siebziger Jahren, in: Konrad H. Jarausch (Hrsg.), Das Ende der Zuversicht? Die siebziger Jahre als Geschichte, Göttingen 2008, S.296-312.

Frank Bösch, Macht und Machtverlust. Die Geschichte der CDU, Stuttgart/München 2002.

Jean Bothorel, Histoire du septennat giscardien, 19 mai 1974-22 mars 1978, Bd. 1: Le Pharaon, Paris 1983.

Patrice Bourdelais, L'Age de la vieillesse, Paris 1997.

Julian Bourg, From Revolution to Ethics. May 1968 and Contemporary French Thought, Montreal 2007.

Christophe Bourseiller, Les maoïstes. La folle histoire des gardes rouges français, Paris 2008. 
Karl Dietrich Bracher, Politik und Zeitgeist. Tendenzen der siebziger Jahre, in: ders./Wolfgang Jäger/Werner Link, Republik im Wandel 1969-1974. Die Ära Brandt, Stuttgart 1986, S. 283406.

Willy Brandt, Über den Tag hinaus. Eine Zwischenbilanz, Hamburg 1974.

Gilles Breton, Libertés publiques et droits de l'homme, Paris 2009.

Bernard Brillant, Les clercs de 68, Paris 2003.

Patrick Bruneteaux, Maintenir l'ordre, Paris 1996.

Günter Buchstab mit Denise Lindsay (Bearb.), Barzel: „Unsere Alternativen für die Zeit der Opposition“. Die Protokolle des CDU-Bundesvorstands 1969-1973, Düsseldorf 2009.

Der Bundesminister für Arbeit und Sozialordnung (Hrsg.), Mitbestimmung - Mitbestimmungs-Gesetz, Montan-Mitbestimmung, Betriebsverfassung, Bonn 1976.

Oldrich Bures, EU Counterterrorism Policy. A Paper Tiger?, Farnham 2011.

Karl Carstens, Erinnerungen und Erfahrungen, hrsg. von Kai von Jena und Reinhard Schmoeckel, Boppard am Rhein 1993.

Peter Chalk, West European Terrorism and Counter-Terrorism. The Evolving Dynamic, Houndmills/London 1996.

Sophie Chauveau, L'économie de la France au 20e siècle, Paris 2000.

Jacques Chevallier, L'État de droit, Paris 1994.

Michael Scott Christofferson, French Intellectuals Against The Left. The Antitotalitarian Moment of the 1970s, New York/Oxford 2004.

Vincent Cloarec/Henry Laurens, Le Moyen-Orient au 20 e siècle, Paris 2010.

Samy Cohen, De Gaulle et Israël. Le sens d'une rupture, in: Élie Barnavi/Saul Friedländer (Hrsg.), La politique étrangère du Général de Gaulle, Paris 1985, S. 192-202.

Samy Cohen/Marie-Claude Smouts (Hrsg.), La politique extérieure de Valéry Giscard d'Estaing, Paris 1985.

Michèle Cotta, Secrets d'État, Bd. 2: 1977-1988, 15 octobre 1977, Paris 2008.

Christopher Daase, Die RAF und der internationale Terrorismus. Zur transnationalen Kooperation klandestiner Organisationen, in: Wolfgang Kraushaar (Hrsg.), Die RAF und der linke Terrorismus, Bd. 2, Hamburg 2006, S. 905-929.

Matthias Dahlke, Demokratischer Staat und transnationaler Terrorismus. Drei Wege zur Unnachgiebigkeit in Westeuropa 1972-1975, München 2011.

Ralf Dahrendorf, Krise der Demokratie? Eine kritische Betrachtung, in: Daniel Frei (Hrsg.), Überforderte Demokratie?, Zürich 1978, S. 55-72.

Dominique Dessertine/Olivier Faure, Combattre la tuberculose, Lyon 1988.

Thierno Diallo, La politique étrangère de Georges Pompidou, Paris 1992.

Démocratie et pauvreté. Du quatrième ordre au quart monde. Actes du colloque de Caen organisé par ATD QUART MONDE, octobre 1989, Paris 1991.

Jürgen Dittberner, Die FDP. Geschichte, Personen, Organisation, Perspektiven. Eine Einführung, Wiesbaden 2005.

Anselm Doering-Manteuffel/Lutz Raphael, Nach dem Boom. Perspektiven auf die Zeitgeschichte seit 1970, Göttingen 2008.

Marnix Dressen, Les établis, la chaîne et le syndicat. Évolution des pratiques, mythes et croyances d'une population d'établis maoïstes, 1968-1982. Monographie d'une usine lyonnaise, Paris 2000.

Denis Duez, L'Union européenne et l'immigration clandestine. De la sécurité intérieure à la construction de la communauté politique, Brüssel 2008.

Abba Eban, Personal Witness. Israel Through My Eyes, New York 1992.

Bernhard Ebbinghaus/JelleVisser, The Societies of Europe: Trade Unions in Western Europe since 1945, London 2000.

Horst Ehmke, Mittendrin. Von der großen Koalition zur Deutschen Einheit, Berlin 1994.

Joachim Samuel Eichhorn, Durch alle Klippen hindurch zum Erfolg. Die Regierungspraxis der ersten Großen Koalition (1966-1969), München 2009.

The Encyclopedia of the Arab-Israeli Conflict. A Political, Social, and Military History, Bd.4: Documents, Santa Barbara 2008.

Jean-Pierre Faugère/Colette Voisin, Le système financier et monétaire international. Crises et mutations, Paris 2005. 
Bernd Faulenbach, Die Siebziger Jahre - ein sozialdemokratisches Jahrzehnt?, in: Archiv für Sozialgeschichte 44 (2004), S. 1-37.

Jean Fourastié, Les trente glorieuses. Ou la révolution invisible de 1945 à 1975, Paris 1979.

La France du XX ${ }^{\mathrm{e}}$ siècle. Documents d'histoire, présentés par Olivier Wieviorka et Christophe Prochasson, Paris 1994.

Robert Frank/Geneviève Dreyfus-Armand/Maryvonne Le Puloch, Marie-Francoise Lévy/Michelle Zancarini-Fournel, Crises et conscience de crise. Les années grises de la fin de siècle, in: Vingtième siècle Nr. 84, 2004, S. 75-82.

Matthias Frese/Julia Paulus/Karl Teppe (Hrsg.), Demokratisierung und gesellschaftlicher Aufbruch. Die sechziger Jahre als Wendezeit der Bundesrepublik, Paderborn 2003.

Jörg Friedrichs, Fighting Terrorism and Drugs. Europe and international police cooperation, London/New York 2008.

Henri Froment-Meurice, Vu du Quai, Paris 1998.

Marc Fumaroli, Les abeilles et les araignées, in: La querelle des anciens et des modernes. XVII XVIII siècles, Paris 2001, S. 55-76.

Albrecht Funk, Das deutsche System der Inneren Sicherheit im Prozeß der Europäisierung, in: Hans-Jürgen Lange (Hrsg.), Staat, Demokratie und Innere Sicherheit in Deutschland, Opladen 2000, S. 291-309.

Alexander Gallus, Zäsuren in der Geschichte der Bundesrepublik, in: Hans-Peter Schwartz, Die Bundesrepublik Deutschland: Eine Bilanz nach 60 Jahren, Köln/Weimar/Wien 2008, S.35-56.

Johan Galtung, Gewalt, Frieden und Friedensforschung, in: Dieter Senghaas (Hrsg.), Kritische Friedensforschung, Frankfurt am Main 1971, S. 55-104.

Jean Garrigues, Les Patrons et la politique de Schneider à Seillière, Paris 2002.

André Gauron, Histoire économique et sociale de la Cinquième République, Bd. 2: Années de rêve, années de crise: 1970-1981, Paris 1988.

André Gauron, Quelques détails sur le plan Fourcade in: Jean-Jacques Becker/Pascal Ory, Crises et alternances 1974-2000, Paris 2002, S. 66-74.

Andrew Geddes, Immigration and European Integration. Beyond fortress Europe?, Manchester/New York 22008.

Alain Geismar/Serge July/Erlyne Morane, Vers la guerre civile, Paris 1969.

Martin H. Geyer, Rahmenbedingungen: Unsicherheit als Normalität, in: Geschichte der Sozialpolitik in Deutschland seit 1945. Hrsg. vom Bundesministerium für Arbeit und Soziales und Bundesarchiv, Bd.6: Bundesrepublik Deutschland 1974-1982. Neue Herausforderungen, wachsende Unsicherheiten. Bandherausgeber: Martin H. Geyer, Baden-Baden 2008, S. 1-109.

Ingrid Gilcher-Holtey, „Die Phantasie an die Macht“. Mai 68 in Frankreich, Frankfurt am Main 1995.

Yves Girard, Un neutron entre les dents, Paris 1997.

Valéry Giscard d'Estaing, Démocatie française, Paris 1976.

Valéry Giscard d'Estaing, L'état de la France, Paris 1981.

Valéry Giscard d'Estaing, Le pouvoir et la vie, Bd. 2, Paris 1991.

Alain Glucksmann, Fascismes: l'ancien et le nouveau, in: Les Temps Modernes, Nr. 310 bis, 1972, S. 266-334.

Boris Gobille, Mai 68, Paris 2008.

Manfred Görtemaker, Geschichte der Bundesrepublik Deutschland. Von der Gründung bis zur Gegenwart, München 1999.

Bernhard Gotto/Horst Möller/Jean Mondot/Nicole Pelletier (Hrsg.), Krisen und Krisenbewusstsein in Deutschland und Frankreich in den 1960er Jahren, München 2012.

Helga Grebing, Willy Brandt. Der andere Deutsche, München 2008.

Pascal Grisé, Georges Pompidou et la modernité. Les tensions de l'innovation 1962-1974, Brüssel 2006.

Herbert Gruhl, Ein Planet wird geplündert. Die Schreckensbilanz unserer Politik, Frankfurt am Main 1975.

Pierre Guillaume, Du désespoir au salut: les tuberculeux aux XIXe et XXe siècle, Paris 1986.

Pierre Guillaume, Histoire sociale de la France au XXe siècle, Paris 1992.

Pierre Guillaume, Un projet, la Nouvelle société, in: Bernard Lachaise/Gilles Le Béguec/JeanFrançois Sirinelli (Hrsg.), Jacques Chaban Delmas en politique, Paris 2007, S. 185-223. 
Pierre Guillaume, Un siècle d'histoire de l'enfance inadaptée. L'OREAG 1889-1989, Paris 1989. Sylvie Guillaume, L'action gouvernementale: la gestion de la fin du septennat, in: Serge Berstein/ Jean-François Sirinelli (Hrsg.), Les années Giscard 1978-1981. Les institutions à l'épreuve?, Paris 2010, S. 57-73.

Sylvie Guillaume, L'avènement des libéraux, in: Jean Garrigues/Sylvie Guillaume/Jean-François Sirinelli (Hrsg.), Comprendre la Ve République, Paris 2010, S. 307-326.

Sylvie Guillaume, La France contemporaine, chronologie commentée (1959-1989), Paris 1991. Sylvie Guillaume, Le petit et moyen patronat, Bordeaux 2004.

Sylvie Guillaume/Pierre Guillaume, Réformes et réformisme dans la France contemporaine, Paris 2012.

Jürgen Habermas, Legitimationsprobleme im Spätkapitalismus, Frankfurt am Main 1973.

Jürgen Habermas, Stichworte zur „Geistigen Situation der Zeit“, Bd. 1, Frankfurt am Main 1979.

Jens Hacke, Staat in Gefahr. Die Bundesrepublik der 1970er Jahre zwischen Legitimationskrise und Unregierbarkeit, in: ders./Dominik Geppert (Hrsg.), Streit um den Staat. Intellektuelle Debatten in der Bundesrepublik 1960-1980, Göttingen 2008, S. 188-206.

Hervé Hamon/Patrick Rotman, Génération, Bd. 2: Les années de poudre, Paris 1988.

Charles-Noël Hardy (Hrsg.), Imaginer l'avenir. Propositions libérales établies par les clubs Perspectives et Réalités, Paris 1972.

Jacques Harstrich, 20 ans de police politique, Paris 1991.

Heinz Gerhard Haupt/Jörg Requate (Hrsg.), Aufbruch in die Zukunft. Die 1960er Jahre zwischen Planungseurphorie und kulturellem Wandel. DDR, CSSR und Bundesrepublik im Vergleich, Weilerswist 2004.

Siegfried Heimann, Die Sozialdemokratische Partei Deutschlands, in: Richard Stöss (Hrsg.), Parteien-Handbuch. Die Parteien der Bundesrepublik Deutschland 1945-1980, Bd. 4, Opladen 1986, S. 2025-2216.

Pierre Jakez Héliaz, Le Cheval d'orgueil, Paris 1975.

Wilhelm Hennis/Peter Kielmansegg/Ulrich Matz (Hrsg.), Regierbarkeit. Studien zu ihrer Problematisierung, 2 Bde., Stuttgart 1977/79.

Ulrich Herbert, Geschichte der Ausländerpolitik in Deutschland, München 2001.

Claudia Hiepel, Willy Brandt und Georges Pompidou. Deutsch-französische Europapolitik zwischen Aufbruch und Krise, München 2012.

Histoire de la France rurale. Sous la direction de Georges Duby, Georges Bertrand, Hugues Neveux, Maurice Agulhon et Michel Gervais, Paris 1975.

Eric Hobsbawn, Age of Extremes. The short twentieth century, 1914-1991, London 1994; deutsche Ausgabe: Das Zeitalter der Extreme. Weltgeschichte des 20. Jahrhunderts, München 1995.

Hans Günter Hockerts, Rahmenbedingungen: Das Profil der Reformära, in: Geschichte der Sozialpolitik in Deutschland seit 1945. Hrsg. vom Bundesministerium für Arbeit und Soziales und Bundesarchiv, Bd. 5: Bundesrepublik Deutschland 1966-1974. Eine Zeit vielfältigen Aufbruchs. Bandherausgeber: Hans Günter Hockerts, Baden-Baden 2006, S. 1-155.

Hans Günter Hockerts/Winfried Süß, Der Wohlfahrtsstaat in einer Zeit vielfältigen Aufbruchs. Zur sozialpolitischen Bilanz der Reformära, in: Hans Günter Hockerts (Hrsg.): Geschichte der Sozialpolitik in Deutschland seit 1945, Bd. 5: Bundesrepublik Deutschland 1966-1974. Eine Zeit vielfältigen Aufbruchs, Baden-Baden 2006, S. 943-962.

Peter Hoeres, Von der „Tendenzwende“ zur „geistig-moralischen Wende“. Konstruktion und Kritik konservativer Signaturen in den 1970er und 1980er Jahren, in: VfZ 61 (2013), S. 93119.

Bruce Hoffman, Terrorismus - der unerklärte Krieg. Neue Gefahren politischer Gewalt, Bonn 2007.

Jens Hohensee, Der erste Ölpreisschock 1973/74. Die politischen und gesellschaftlichen Auswirkungen der arabischen Erdölpolitik auf die Bundesrepublik Deutschland und Westeuropa, Stuttgart 1996.

Robert Horvath, „The Solzhenitsyn Effect“. East European Dissidents and the Demise of the Revolutionary Privilege, in: Human Rights Quarterly 29 (2007), S. 879-907.

François Hourmant, Le désenchantement des clercs. Figures de l'intellectuel dans l'après-mai 1968, Paris 1998. 
Jim House/Neil Macmaster, Paris 1961: Algerians, State Terror, and Memory, Oxford 2006.

Stefan Hradil/Holger Schmidt, Angst und Chancen. Zur Lage der gesellschaftlichen Mitte aus soziologischer Sicht, in: Herbert-Quandt-Stiftung (Hrsg.), Zwischen Erosion und Erneuerung. Die gesellschaftliche Mitte in Deutschland. Ein Lagebericht, Frankfurt am Main 2007, S. $163-225$.

Ronald Inglehart, The Silent Revolution. Changing Values and Political Styles among Western Publics, Princeton 1977.

Wolfgang Jäger, Die Innenpolitik der sozial-liberalen Koalition 1969-1974, in: Karl Dietrich Bracher/Wolfgang Jäger/Werner Link, Republik im Wandel 1969-1974. Die Ära Brandt, Stuttgart 1986, S. 13-160.

Wolfgang Jäger/Werner Link, Republik im Wandel 1974-1982. Die Ära Schmidt, Stuttgart 1987.

Martin Jander, Isolation. Zu den Haftbedingungen der RAF-Gefangenen, in: Wolfgang Kraushaar (Hrsg.), Die RAF und der linke Terrorismus, Bd. 2, Hamburg 2006, S. 973-983.

Konrad H. Jarausch (Hrsg.), Das Ende der Zuversicht? Die siebziger Jahre als Geschichte, Göttingen 2008.

Konrad H. Jarausch, Verkannter Strukturwandel. Die siebziger Jahre als Vorgeschichte der Probleme der Gegenwart, in: ders. (Hrsg.), Das Ende der Zuversicht? Die siebziger Jahre als Geschichte, Göttingen 2008, S. 9-26.

Fabien Jobard, Ce que Mai fit à la police, in: Philippe Artières/Michelle Zancarini-Fournel (Hrsg.), Les années 68. Une histoire collective (1962-1981), Paris 2008, S. 577-582.

Michel Jobert, Mémoires d'avenir, Paris 1974.

Samir Kassir/Farouk Mardam-Bey, Itinéraires de Paris à Jérusalem. La France et le conflit israélo-arabe, Bd. 2: 1958-1991, Paris 1993.

Wolther von Kiersitzky (Bearb.), Mehr Demokratie wagen. Innen- und Gesellschaftspolitik 1966-1974 (= Willy Brandt. Berliner Ausgabe; Bd. 7), Bonn 2001.

Henry Kissinger, Years of Renewal, New York 1999.

Wilhelm Knelangen, Das Politikfeld innere Sicherheit im Integrationsprozess. Die Entstehung einer europäischen Politik der inneren Sicherheit, Opladen 2001.

Franz König, Soziallehre und Kirche, Wien 1977.

Wolfgang Kraushaar, Achtundsechzig. Eine Bilanz, Berlin 2006.

Wolfgang Kraushaar, 1968 als Mythos, Chiffre und Zäsur, Hamburg 2000.

Wolfgang Kraushaar, Der nicht erklärte Ausnahmezustand. Staatliches Handeln während des sogenannten Deutschen Herbstes, in: ders. (Hrsg.), Die RAF und der linke Terrorismus, Bd. 2, Hamburg 2006, S. 1011-1025.

Martin Kriele, Legitimationsprobleme in der Bundesrepublik, München 1977.

Gerhard Kunnert, Touristen aus EU-Drittstaaten als potenzielle Terroristen? Ein kritischer Blick auf Ursprünge, Entwicklung und aktuelle Tendenzen der EU-Außengrenzpolitik, in: Michael Gehler/Andreas Pudlat (Hrsg.), Grenzen in Europa, Hildesheim u. a. 2009, S. 211-268.

Paul-Marie de La Gorce, La politique arabe du Général de Gaulle, in: Élie Barnavi/Saul Friedländer, La politique étrangère du Général de Gaulle, Paris 1985, S. 179-191.

Peter Laemmle, Büchners Schatten. Zur Rezeption von Peter Schneiders Lenz, in: Akzente, Heft 5, Oktober 1974, S. 469-478.

Markus Lammert, Die französische Linke, der Terrorismus und der „repressive Staat“ in der Bundesrepublik in den 1970er Jahren, in: VfZ 59 (2011), S. 533-560.

Henry Laurens, La question de, Bd. 4: 1967-1982. Le rameau d'olivier et le fusil du combattant, Paris 2011.

Karl Lauschke, Mehr Demokratie in der Wirtschaft. Die Entstehungsgeschichte des Mitbestimmungsgesetzes von 1976, Bd. 1, Düsseldorf 2006.

Sandra Lavenex, The Europeanisation of Refugee Policies. Between human rights and internal security, Aldershot 2001.

Sandra Lavenex, Länderprofil Europäische Union, in: focus MIGRATION, Nr. 17, März 2009.

Jean-Pierre Lefebvre, La traversée de la neige, préface, in: Georg Büchner, Lenz. Nouvelle, suivi de Monsieur L..., de Jean Frédéric Oberlin et de Le Dialogue dans la montagne, de Paul Celan, Paris 2007, S. 7-19.

Jean-Pierre Le Goff, Mai 68. L'héritage impossible, Paris 1998. 
Paul Legoll, Charles de Gaulle et Konrad Adenauer. La cordiale entente, Paris 2004.

Michel Lelart, Le système monétaire international, Paris 2003.

Emmanuel Le Roy Ladurie, Montaillou, village occitan de 1294 à 1324, Paris 1975.

Ludger Lindlar, Das missverstandene Wirtschaftswunder. Westdeutschland und die westeuropäische Nachkriegsprosperität, Tübingen 1997.

Werner Link, Außen- und Deutschlandpolitik in der Ära Schmidt 1974-1982, in: ders./Wolfgang Jäger, Republik im Wandel 1974-1982. Die Ära Schmidt, Stuttgart 1987, S. 273-432.

Peter Lösche/Franz Walter, Die FDP. Richtungsstreit und Zukunftszweifel, Darmstadt 1996.

Burkhart Lutz, Der kurze Traum immerwährender Prosperität. Eine Neuinterpretation der industriell-kapitalistischen Entwicklung im Europa des 20. Jahrhunderts, Frankfurt am Main 1989.

Jean-François Lyotard, La condition postmoderne. Rapport sur le savoir, Paris 1979.

Sabine Manke, Brandt anfeuern. Das Misstrauensvotum 1972 in Bürgerbriefen an den Bundeskanzler. Ein kulturwissenschaftlicher Beitrag zu modernen Resonanz- und Korrespondenzphänomenen, Marburg 2008.

Raymond Marcellin, L’ordre public et les groupes révolutionnaires, Paris 1969.

Herbert Marcuse, Der eindimensionale Mensch. Studien zur Ideologie der fortgeschrittenen Industriegesellschaft, München 1994.

Herbert Marcuse, Repressive Toleranz, in: ders./Robert Paul Wolff/Barrington Moore, Kritik der reinen Toleranz, Frankfurt am Main 1966, S. 91-128.

Margaret Mead, Le fossé des générations, Paris 1971.

Donella H. Meadows/Dennis L. Meadows/Jørgen Randers/William W. Behrens III, Die Grenzen des Wachstums. Bericht des Club of Rome zur Lage der Menschheit, Stuttgart 1972.

Golda Meir, Mein Leben, Hamburg 1975.

Thomas Mergel, Krisen als Wahrnehmungsphänomene, in: ders. (Hrsg.), Krisen verstehen. Historische und kulturwissenschaftliche Annäherungen, Frankfurt am Main 2010, S. 9-21.

Thomas Mergel, Propaganda nach Hitler. Eine Kulturgeschichte des Wahlkampfs in der Bundesrepublik 1949-1990, Göttingen 2010.

Gabriele Metzler, Staatsversagen und Unregierbarkeit in den siebziger Jahren?, in: Konrad H. Jarausch (Hrsg.), Das Ende der Zuversicht? Die siebziger Jahre als Geschichte, Göttingen 2008, S. 234-261.

Hélène Miard-Delacroix, Deutsch-französische Geschichte, Bd.11: Im Zeichen der europäischen Einigung. 1963 bis in die Gegenwart, Darmstadt 2011, französische Ausgabe: Le défi européen, de 1963 à nos jours, Villeneuve d'Ascq 2011.

Albert Michel (Hrsg.), Raymond Barre. Un homme singulier dans la politique française, Paris 2010.

Alf Mintzel, Geschichte der CSU. Ein Überblick, Opladen 1977.

Alf Mintzel, Die Christlich-Soziale Union, in: Richard Stöss (Hrsg.), Parteien-Handbuch. Die Parteien der Bundesrepublik Deutschland 1945-1980, Bd. 2, Opladen 1986, S. 661-718.

Valsamis Mitsilegas/Jörg Monar/Wyn Rees, The European Union and Internal Security. Guardian of the People?, Basingstoke u. a. 2003.

Jörg Monar, Die Vertragsreformen von Lissabon in den Bereichen Inneres und Justiz: verstärkte Handlungsfähigkeit, Kontrolle und Differenzierung, in: Integration 32 (2009), S. 379-388.

Jean Mondot, Mauriac, séismographe des relations franco-allemandes des années 1960?, in: Bernhard Gotto/Horst Möller/Jean Mondot (Hrsg.), Krisen und Krisenbewusstsein in Deutschland und Frankreich in den 1960er Jahren, München 2012, S. 205-213.

Jean Monneret, La phase finale de la guerre d'Algérie, Paris u. a. 2010.

Daniela Münkel, John F. Kennedy - Harold Wilson - Willy Brandt: „Modernes“ Image für moderne Zeiten, in: dies./Lu Seegers (Hrsg.), Medien und Imagepolitik im 20. Jahrhundert. Deutschland, Europa, USA, Frankfurt am Main/New York 2008, S. 25-47.

Daniela Münkel, Politiker-Image und Wahlkampf. Das Beispiel Willy Brandt: vom „deutschen Kennedy“ zum „deutschen Helden“, in: Bernd Weisbrod (Hrsg.), Die Politik der Öffentlichkeit - die Öffentlichkeit der Politik. Politische Medialisierung in der Geschichte der Bundesrepublik, Göttingen 2003, S. 55-76.

Daniela Münkel, Willy Brandt und die „vierte Gewalt“. Politik und Massenmedien in den 50er bis 70er Jahren, Frankfurt/New York 2005. 
Oswald von Nell-Breuning, Gerechtigkeit und Freiheit. Grundzüge katholischer Soziallehre, München 1985.

Oswald von Nell-Breuning, Mitbestimmung, Landshut 1950.

Karlheinz Niclauß, Kontroverse Deutschlandpolitik. Die politische Auseinandersetzung in der Bundesrepublik Deutschland über den Grundlagenvertrag mit der DDR, Frankfurt am Main 1979.

Elisabeth Noelle/Peter Neumann (Hrsg.), Jahrbuch der öffentlichen Meinung 1968-1973, Allensbach/Bonn 1974.

Eva Oberloskamp, Das Olympia-Attentat 1972. Politische Lernprozesse im Umgang mit dem transnationalen Terrorismus, in: VfZ 60 (2012), S. 321-352.

Kurt Oesterle, Stammheim. Die Geschichte des Vollzugsbeamten Horst Bubeck, Tübingen 2003.

Dieter Paas, Frankreich: Der integrierte Linksradikalismus, in: Henner Hess/Martin Moerings/ Dieter Paas/Sebastian Scheerer/Heinz Steinert, Angriff auf das Herz des Staates. Soziale Entwicklung und Terrorismus, Frankfurt am Main 1988, S. 169-278.

Erwin Panofsky, Die Renaissancen der europäischen Kunst, Frankfurt am Main 1979.

Parti Communiste Français, Vivre libre. Projet de déclaration des libertés soumis à la discussion des Français, Paris 1975.

Laurence Pellegrini, Regards croisées: Valéry Giscard d'Estaing vu par Die Zeit et Helmut Schmidt vu par Le Nouvel Observateur, in: Allemagne d'aujourd'hui, Nr. 191, 2010, S. 135-147.

Pauline Peretz, La France et la guerre du Kippour, in: Revue d'Histoire diplomatique 120 (2006), Heft 2, S. 143-156.

Georg Picht, Die deutsche Bildungskatastrophe. Analyse und Dokumentation, Olten 1964.

Petra Platen, Zwischen Dableiben und Verschwinden. Zur Kontinuität im Werk von Peter Schneider, München 2006.

Presse- und Informationsamt der Bundesregierung (Hrsg.), Bundeskanzler Brandt. Reden und Interviews, 2 Bde., Melsungen 1971/1973.

Gideon Rafael, Destination Peace. Three Decades of Israeli Foreign Policy. A Personal Memoir, London 1981.

Thomas Raithel/Andreas Rödder/Andreas Wirsching (Hrsg.), Auf dem Weg in eine neue Moderne? Die Bundesrepublik Deutschland in den siebziger und achtziger Jahren, München 2009.

Maurice Rajsfus, Mai 68. Sous les pavés, la répression (mai 1968-mars 1974), Paris 1998.

Pierre Razoux, L'affaire des vedettes de Cherbourg. Pourquoi Paris a laissé faire, in: Historia, novembre 2000, S. 36-40.

René Rémond, Frankreich im 20. Jahrhundert. Zweiter Teil: 1958 bis zur Gegenwart, Stuttgart 1995.

Gerhard A. Ritter/Merith Niehuss, Wahlen in Deutschland 1946-1991. Ein Handbuch, München 1991.

Michel Rocard, Si la Gauche savait. Entretiens avec Georges-Marc Benamou, Paris 2005.

Andreas Rödder, Die Bundesrepublik Deutschland 1969-1990, München 2004.

Anne Rohstock, Von der „Ordinarienuniversität“ zur „Revolutionszentrale“? Hochschulreform und Hochschulrevolte in Bayern und Hessen 1957-1976, München 2010.

Jan-Dirk Rosche, Katholische Soziallehre und Unternehmensordnung, Paderborn u. a. 1988.

François de Rose, La France et la défense de l'Europe, Paris 1976.

Miriam Rosman, La France et Israël 1947-1970. De la création de l'État d'Israël au départ des vedettes de Cherbourg, Paris 2009.

Eric Roussel, Georges Pompidou, Paris 1984.

Serge Savoie, RG. La traque d'Action Directe, Paris 2011.

Axel Schildt, Die Sozialgeschichte der Bundesrepublik Deutschland bis 1989/90, München 2007.

Axel Schildt/Karl Christian Lammers/Detlef Siegfried (Hrsg.), Dynamische Zeiten. Die 60er Jahre in den beiden deutschen Gesellschaften, Hamburg 2000.

Kay Schiller/Christopher Young, The 1972 Munich Olympics and the Making of Modern Germany, Berkeley u. a. 2010.

Boris Schilmar, Der Europadiskurs im deutschen Exil 1933-1945, München 2004. 
Peter Schindler, Datenhandbuch zur Geschichte des Deutschen Bundestages 1949 bis 1999. Gesamtausgabe in drei Bänden. Eine Veröffentlichung der Wissenschaftlichen Dienste des Deutschen Bundestages, Baden-Baden 1999.

Helmut Schmidt, Außer Dienst. Eine Bilanz, München 2008.

Helmut Schmidt, Weggefährten. Erinnerungen und Reflexionen, Berlin 1996.

Ute Schmidt, Die Christlich Demokratische Union Deutschlands, in: Richard Stöss (Hrsg.), Parteien-Handbuch. Die Parteien der Bundesrepublik Deutschland 1945-1980, Opladen 1986, S. 490-660.

Dominique Schnapper, L’Europe des immigrés, Paris 1992.

Peter Schneider, Ansprachen, Berlin 1980 [Erstausgabe 1970].

Peter Schneider, Antwort an einen anonymen Kritiker, in: ders., Atempause. Versuch, meine Gedanken über Literatur und Kunst zu ordnen, Reinbek bei Hamburg 1977, S. 202-203.

Peter Schneider, Lenz. Eine Erzählung, Berlin 1973.

Peter Schneider, Rebellion und Wahn. Mein '68, Köln 2008.

Peter Schneider, Rede an die deutschen Leser und ihre Schriftsteller [1968], in: ders., Ansprachen, Berlin 1980 [Erstausgabe 1970], S. 29-38.

Peter Schneider, ... schon bist du ein Verfassungsfeind. Das unerwartete Anschwellen der Personalakte des Lehrers Kleff, Berlin 1975.

Peter Schneider, „Wir haben Fehler gemacht“, in: ders., Ansprachen, Berlin 1980 [Erstausgabe 1970], S. 7-14.

Ralf Schnell, Die Literatur der Bundesrepublik. Autoren, Geschichte, Literaturbetrieb, Stuttgart 1986.

Wulf Schönbohm, Die CDU wird moderne Volkspartei. Selbstverständnis, Mitglieder, Organisation und Apparat 1950-1980, Stuttgart 1985.

Hans-Peter Schwarz, Der Ort der Bundesrepublik in der deutschen Geschichte, Opladen 1996.

Sabine Seggelke, Der französische Präsident Valéry Giscard d'Estaing und die Massenmedien. Vom „Kennedy à la française“ zum skandalierten „Monarchen“, Remscheid 2004.

Luciano Segreto, La politique américaine et la crise du système monétaire international (19681973). Vers le flottement général des monnaies, in: Éric Bussière (Hrsg.), Georges Pompidou face à la mutation économique de l'Occident 1969-1974, Paris 2003, S. 31-38.

Jean-François Sirinelli, Désenclaver l'histoire. Nouveaux regards sur le XX $\mathrm{X}^{\mathrm{e}}$ siècle français, Paris 2013.

Jean-François Sirinelli, Deux intellectuels dans le siècle, Sartre et Aron, Paris 1995.

Jean-François Sirinelli (Hrsg.), Dictionnaire historique de la vie politique française au XX siècle, Paris 2003.

Jean-François Sirinelli, L'histoire politique à l'heure du transnational turn: l'agora, la Cité, le monde... et le temps, in: Revue historique No 658 (2011).

Jean-François Sirinelli, Les vingt décisives. Le passé proche de notre avenier 1965-1985, Paris 2007.

Thomas Skelton Robinson, Im Netz verheddert. Die Beziehungen des bundesdeutschen Linksterrorismus zur Volksfront für die Befreiung Palästinas (1969-1980), in: Wolfgang Kraushaar (Hrsg.), Die RAF und der linke Terrorismus, Bd. 2, Hamburg 2006, S. 828-904.

Hartmut Soell, Helmut Schmidt. Macht und Verantwortung, München 2008.

Isabelle Sommier, La violence politique et son deuil. L'après 68 en France et en Italie, Rennes 2008.

Georges-Henri Soutou, L'Alliance incertaine. Les rapports politico-stratégiques franco-allemands, 1954-1996, Paris 1996.

Georges-Henri Soutou, L'anneau et les deux triangles: les rapports franco-allemands dans la politique européenne et mondiale de 1974 à 1981, in: Serge Berstein/Jean-François Sirinelli (Hrsg.), Les années Giscard. Valéry Giscard d'Estaing et l'Europe 1974-1981, Paris 2006, S. 107-123.

Georges-Henri Soutou, Le Président Pompidou et les relations entre les Etats-Unis et l'Europe, in: Journal of European Integration History 6 (2000), Nr. 2, S. 111-146.

Georges-Henri Soutou, Les présidents Charles de Gaulle et Georges Pompidou et les débuts de la coopération politique européenne: du plan Fouchet au plan Fouchet light, in: Relations Internationales, Nr. 140, 2009, S. 3-19. 
Georges-Henri Soutou, Mitläufer der Allianz? Frankreich und der NATO-Doppelbeschluss, in: Philipp Gassert/Tim Geiger/Hermann Wentker (Hrsg.), Zweiter Kalter Krieg und Friedensbewegung. Der NATO-Doppelbeschluss in deutsch-deutscher und internationaler Perspektive, München 2011, S.363-376.

Georges-Henri Soutou, Notice sur la vie et les travaux de Raymond Barre (1924-2007), lue lors de la séance du 12 avril 2010, Paris 2010.

Jean-Marie Soutou, Un diplomate engagé. Mémoires 1939-1979, Paris 2011.

Torsten Stein/Christian Meiser, Die Europäische Union und der Terrorismus, in: Die FriedensWarte 76 (2001), S. 33-54.

Ulf G. Sternberger (Hrsg.), „In der Strafsache gegen Andreas Baader, Ulrike Meinhof, Jan-Carl Raspe, Gudrun Ensslin wegen Mordes u.a.“. Dokumente aus dem Prozeß, Frankfurt am Main 1977.

Georg Stötzel/Martin Wengeler/Karin Böke, Kontroverse Begriffe. Geschichte des öffentlichen Sprachgebrauchs in der Bundesrepublik Deutschland, Berlin 1995.

Winfried Süß, „Wer aber denkt für das Ganze?“ Aufstieg und Fall der ressortübergreifenden Planung im Bundeskanzleramt, in: Matthias Frese/Julia Paulus/Karl Teppe (Hrsg.): Demokratisierung und gesellschaftlicher Aufbruch. Die sechziger Jahre als Wendezeit der Bundesrepublik, Münster 2003, S.349-377.

Tim Szatkowski, Karl Carstens. Eine politische Biographie, Köln u. a. 2007.

Volkmar Theobald (Hrsg.),Von der Europäischen Union zur „Europäischen Sicherheitsunion“? Die Gemeinsame Politik der Inneren Sicherheit in der EU, Berlin 1997.

Dietrich Thränhardt, Geschichte der Bundesrepublik Deutschland, Frankfurt am Main 1996.

Angelika Timm, Israel. Geschichte eines Staates seit seiner Gründung, 3., durchges. und erw. Aufl., Bonn 1998.

Maurice Vaïsse, La grandeur. Politique étrangère du général de Gaulle 1958-1969, Paris 1998.

Jean-Marie Verdier, Le Rapport Sudreau, in: Revue internationale de droit privé 28 (1976), S. 771-783.

Jeremy Waddington, La syndicalisation en Europe. Étendue du problème et éventail des réponses proposées par les syndicats. Document de travail pour l'Université d'été de la CES/ETUIREHS des dirigeants syndicaux européens, Florence 1-2 juillet 2005.

Matthias Wächter, Helmut Schmidt und Valéry Giscard d'Estaing. Auf der Suche nach Stabilität in der Krise der 70er Jahre, Bremen 2011.

Neil Walker, The accountability of European police institutions, in: European Journal on Criminal Policy and Research 1 (1993), S. 34-52.

Franz Walter, Die SPD. Vom Proletariat zur Neuen Mitte, Berlin 2002.

Wolfram Weimer, Deutsche Wirtschaftsgeschichte. Von der Währungsreform bis zum Euro, Hamburg 1998.

Albrecht Wellmer, Terrorismus und Gesellschaftskritik, in: Jürgen Habermas (Hrsg.), Stichworte zur „Geistigen Situation der Zeit“, Bd. 1, Frankfurt am Main 1979., S. 265-293.

August Winkler, Der lange Weg nach Westen, Bd. 2: Deutsche Geschichte vom „Dritten Reich“ bis zur Wiedervereinigung, München 2000.

Andreas Wirsching, Abschied vom Provisorium. Geschichte der Bundesrepublik Deutschland 1982-1990, Stuttgart 2006.

Edgar Wolfrum, Die Bundesrepublik Deutschland 1949-1990, Stuttgart 2005.

Edgar Wolfrum, Die geglückte Demokratie. Geschichte der Bundesrepublik Deutschland von ihren Anfängen bis zur Gegenwart, Stuttgart 2006.

Richard Wolin, The Wind from the East. French Intellectuals, the Cultural Revolution and the Legacy of the 1960s, Princeton/Oxford 2010.

David Yost, La France et la sécurité européenne, Paris 1985.

Michelle Zancarini-Fournel, Changer le monde et changer sa vie, in: dies./Philippe Artières (Hrsg.), Les années 68. Une histoire collective (1962-1981), Paris 2008, S. 403-443.

Francis Zamponi, Raymond Marcellin, le Fouché breton, in: Robert Faligot/Jean Guisnel (Hrsg.), Histoire secrète de la Ve République, Paris 2006. 


\section{Abkürzungen}

AA

AAPD

AdsD

AFEP

AIE

AN

Anm.

ASSEDIC

ATD

Aufl.

BA

Bd./Bde.

bearb.

Bearb.

BfV

BGBl

BK

BKA

$\mathrm{BM}$

BMI

BSN

BT-Drs.

bzw.

ca.

CAP

CDD

CDI

CDU

CEE

cf.

CFDT

CFTC

CGC

CGE

CGPME

CGT

CGT-FO

$\mathrm{CNI}$

CNPF

CRS

CSSR

CSU

d. h.
Auswärtiges Amt

Akten zur Auswärtigen Politik der Bundesrepublik Deutschland Archiv der sozialen Demokratie

Association française des entreprises privées

Agence internationale de l'énergie

Archives nationales

Anmerkung

Association pour l'emploi dans l'industrie et le commerce

Agir Tous ensemble pour la Dignité

Auflage

Bundesarchiv

Band/Bände

bearbeitet

Bearbeiter/in

Bundesamt für Verfassungsschutz

Bundesgesetzblatt

Bundeskanzler

Bundeskanzleramt

Bundesminister

Bundesministerium des Innern

Boussois-Souchon-Neuvecelle

Bundestagsdrucksache

Beziehungsweise

circa

Centre d'Analyse et de Prévision

Contrats à durée déterminée

Contrats à durée indéterminée

Christlich-Demokratische Union Deutschlands

Communauté économique européenne

confer

Confédération française démocratique du travail

Confédération française des travailleurs chrétiens

Confédération générale des cadres

Compagnie Générale des Eaux

Confédération générale des petites et moyennes entreprises

Confédération générale du travail

Confédération générale du travail - Force ouvrière

Centre national des indépendants

Conseil national du patronat français

Compagnies républicaines de sécurité

Tschechoslowakische Sozialistische Republik

Christlich-Soziale Union in Bayern

das heißt 


\begin{tabular}{|c|c|}
\hline DDR & Deutsche Demokratische Republik \\
\hline ders. & \\
\hline DGB & Deutscher Gewerkschaftsbund \\
\hline dies. & dieselbe \\
\hline DM & Deutsche Mark \\
\hline Dok. & Dokument \\
\hline durchges. & durchgesehen \\
\hline DVD & Digital Versatile Disc \\
\hline Ebd. & Ebenda \\
\hline éd. & éditeur \\
\hline EDF & Électricité de France \\
\hline EDV & Elektronische Datenverarbeitung \\
\hline EG & Europäische Gemeinschaft \\
\hline ENA & École Nationale d'Administration \\
\hline entst. & entstanden \\
\hline EPR & European Pressurized Water Reactor \\
\hline EPZ & Europäische Politische Zusammenarbeit \\
\hline erw. & erweitert \\
\hline et al. & et alii \\
\hline EU & Europäische Union \\
\hline EWG & Europäische Wirtschaftsgemeinschaft \\
\hline f./ff. & folgend/fortfolgend \\
\hline FAR & Fraction Armée Rouge \\
\hline fr. & français \\
\hline FDP & Freie Demokratische Partei \\
\hline FMI & Fonds monétaire international \\
\hline FU & Freie Universität \\
\hline FO & Foreign Office \\
\hline FU & Freie Universität \\
\hline GG & Grundgesetz \\
\hline GIP & Groupe d'information sur les prisons \\
\hline GP & Gauche Prolétarienne \\
\hline GRECE & Groupement de recherches et d'études pour la civilisation européenne \\
\hline GSG 9 & Grenzschutzgruppe 9 \\
\hline Hrsg. & Herausgeber(in) \\
\hline Ibid. & Ibidem \\
\hline id. & idem \\
\hline IEP & Institut d'Études Politiques \\
\hline IFOP & Institut français d'opinion publique \\
\hline IfZ & Institut für Zeitgeschichte \\
\hline IMK & Innenministerkonferenz \\
\hline insbes. & insbesondere \\
\hline INSEE & Institut de la Statistique et des Etudes Economiques \\
\hline IVG & Interruption volontaire de grossesse \\
\hline JCR & Jeunesse communiste révolutionnaire \\
\hline JFK & John F. Kennedy \\
\hline
\end{tabular}


JORF Journal Officiel de la République Française

Kap. Kapitel

KSZE Konferenz über Sicherheit und Zusammenarbeit in Europa

KPdSU Kommunistische Partei der Sowjetunion

KVAE Konferenz über vertrauensbildende Maßnahmen und Abrüstung in Europa

M. Monsieur

MAE Archives du Ministère des Affaires étrangères

MBFR Mutual and Balanced Force Reductions

MD Ministerialdirektor

MDg Ministerialdirigent

Medef Mouvement des entreprises de France

MR Ministerialrat

MRP Mouvement républicain populaire

$\mathrm{n}^{\circ} \quad$ numéro

NATO North Atlantic Treaty Organization

$\mathrm{n}^{\text {lle }} \quad$ nouvelle

$\mathrm{N}+\mathrm{N} \quad$ Neutrale und nicht Pakt-gebundene Staaten

NPD Nationaldemokratische Partei Deutschlands

Nr. Nummer

o. D. ohne Datum

OAPEC Organization of Arab Petroleum Exporting Countries

OAS Organisation armée secrète

ONU Organisation des Nations Unies

op. cit. opere citato

OPEP Organisation des pays exportateurs de pétrole

OREAG Orientation Rééducation Enfants Adolescents Gironde

ORR Oberregierungsrat

ORFT Office de radiodiffusion-télévision française

OTAN Organisation du Traité de l'Atlantique du Nord

p./pp. page/pages

PA-AA Politisches Archiv des Auswärtigen Amts

PCF Parti communiste français

PDG Président-directeur général

PESC Politique étrangère et de sécurité commune

PIB Produit intérieur brut

PME Petites et moyennes entreprises

PS Parti socialiste

PSU Parti socialiste unifié

RAF Rote Armee Fraktion

RDA République démocratique allemande

RFA République fédérale d'Allemagne

RG (Direction centrale des) Renseignements généraux

RPR Rassemblement pour la République

S. Seite

S.C. Res. United Nations Security Council Official Resolutions 
s. o. siehe oben

SACEUR Supreme Allied Commander Europe

SALT Strategic Arms Limitation Talks

SFIO Section française de l'Internationale ouvrière

SME Système monétaire européen

SNCF Société Nationale des Chemins de fer française

sog. sogenannt

SPD Sozialdemokratische Partei Deutschlands

ss. suivantes

StGB Strafgesetzbuch

t. tome

TF 1 Télévision Française 1

TGV Train à grande vitesse

TNP Traité de non-prolifération nucléaire

TREVI Terrorisme, Radicalisme, Extrémisme, Violence Internationale

TVA Taxe sur la valeur ajoutée

u. a. unter anderem

UDF Union pour la Démocratie Française

UN United Nations

UNEDIC Union nationale interprofessionnelle pour l'emploi dans l'industrie et le commerce

UNO United Nations Organization

URSS Union des Républiques Socialistes Soviétiques

USA/U.S. United States of America

v. von

VBM Vertrauensbildende Maßnahmen

Verf. $\quad$ Verfasser

VfZ Vierteljahrshefte für Zeitgeschichte

Vgl. Vergleiche

WBA Willy-Brandt-Archiv

WVO Warschauer Vertragsorganisation

z.B. zum Beispiel 



\section{Personenregister}

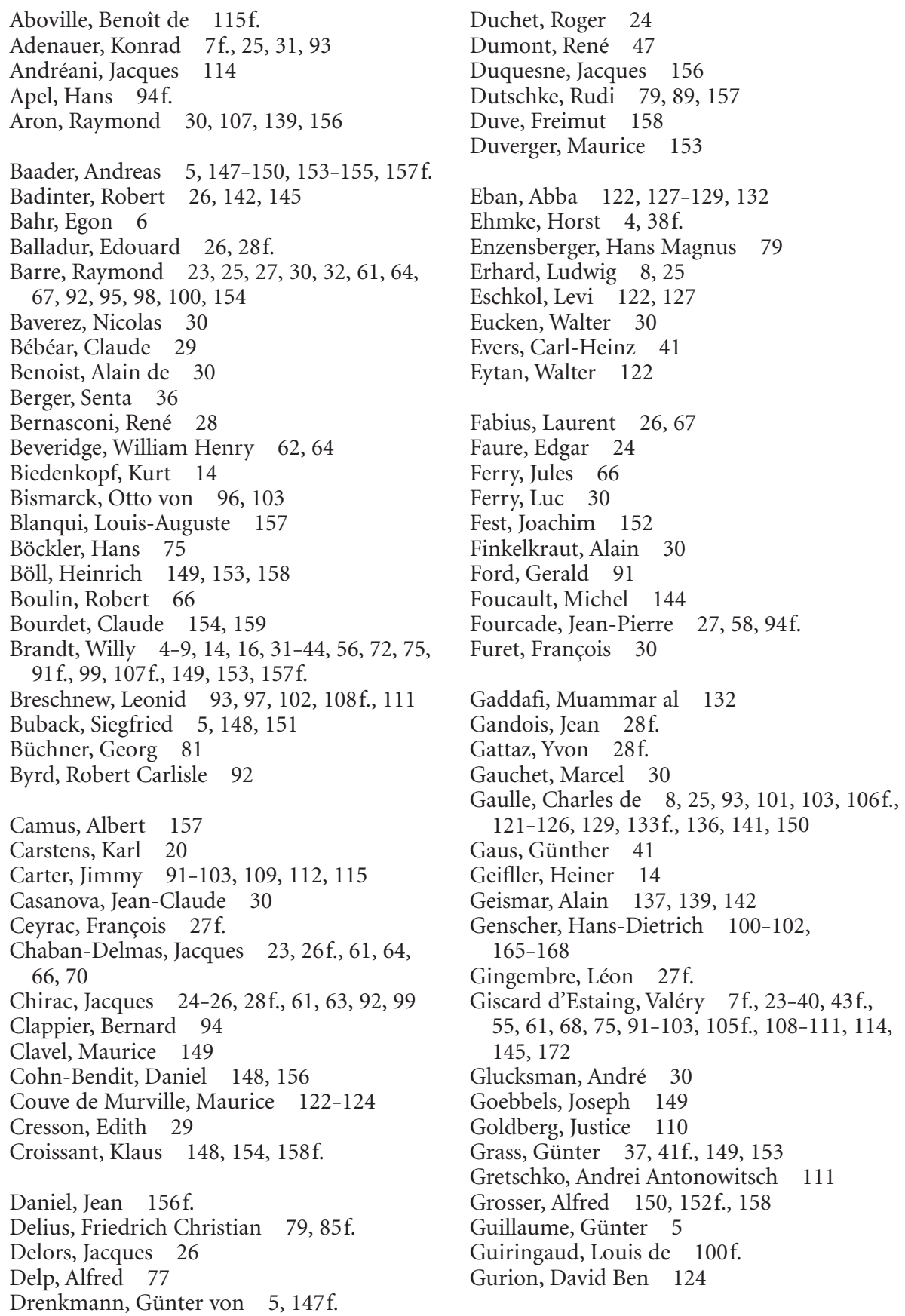


Habermas, Jürgen $\quad 13,79,154$

Haby, René 39

Handke, Peter 79, 87

Harpprecht, Klaus 41

Heinemann, Gustav 20, 158, 177

Hitler, Adolf $\quad 33,93,149,152$

Hollande, François 30

Huré, Francis 132

Jarring, Gunnar 126-129, 133

Jaruzelski, Wojciech 116

Jobert, Michel 130, 132f., 181

Jospin, Lionel 26, 63

Juppé, Alain 29, 63

Kampelmann, Max 115

Kennedy, John F. 34f., 182

Kiesinger, Kurt Georg 6-8

Kiesinger, Kurt-Georg 39

King, Martin Luther 89

Kissinger, Henry 91, 106, 181

Kleist, Heinrich von 157

Klemperer, Victor 88

Kohl, Helmut $\quad$ 5-7, 9, 14, 19, 20, 44, 99

Korsch, Karl 157

Kurras, Karl-Heinz 88

Larguiller, Arlette 155

Laroque, Pierre 61, 64

Léotard, François 29

Lévy, Bernard Henri 30, 146

Lipkowski, Jean de 127

Lorenz, Peter 5, 151

Luxemburg, Rosa 157, 163

Madelin, Alain $\quad 23,26,29$

Maihofer, Werner $\quad 149,173$

Mao Tse-Tung 82, 157

Marcellin, Raymond 135f., 138-142, 146, $167 \mathrm{f}$.

Marcuse, Herbert $\quad 89,153$

Marseille, Jacques 30

Marx, Karl 30, 81, 157

Massu, Jacques 136

Mauroy, Pierre 67

Mead, Margaret 49

Meinhof, Ulrike $\quad$ 147-149, 151f., 156

Meins, Holger 147

Meir, Golda 126-129, 131

Mélenchon, Jean-Luc 30

Mende, Erich 15

Meroz, Yohanan 122

Méry, Guy 98

Messmer, Pierre 61, 132f.

Minc, Alain 30
Mitterrand, François 23, 25f., 28f., 35, 61, 67, 99, 131, 145, 153f., 159

Moreau, Yves 154

Morel, Pierre 117

Müller-Armack, Alfred 30

Nasser, Gamal Abdel $\quad$ 122, 126

Nell-Breuning, Oswald von 77

Nixon, Richard 52, 106, 130

Olcay, Osman 127

Ossietzky, Carl von 149

Pannekoek, Anton 157

Pinay, Antoine 24, 27, 29

Poher, Alain 143

Poincaré, Raymond 27

Pompidou, Georges $\quad 8 f ., 31,33,52,61,67$, $75,92,105-109,111,118,121,126$, 130-136, 168, 173

Poniatowski, Michel 138

Ponto, Jürgen 5,151

Quiliès, Paul 28

Rafael, Gideon 122

Reagan, Ronald 26, 29, 103

Rebuffel, Lucien 29

Rémond, René $\quad 24,40,183$

Revel, Jean-François 30

Riboud, Antoine 27

Robin, Gabriel 91, 93, 109, 111

Rocherau de la Sablière, Bertrand Edmond 124

Rogers, William Pierce $\quad 126,128 \mathrm{f}$.

Röpke, Wilhelm 30

Rosanvallon, Pierre 30

Roux, Ambroise $28 \mathrm{f}$.

Sapir, Pinchas 133

Sarkozy, Nicolas 26, 30

Sartre, Jean-Paul 29f., 135, 137, 144f., $147-150$

Savary, Alain 67

Scheel, Walter $\quad 6,8,15,20,34,42,108,166$

Schiller, Friedrich $\quad 81,157,164,183$

Schleyer, Hanns Martin $\quad 5,19,89,151,153 f$.

Schmidt, Helmut 4-9, 15, 21, 31f., 35, 39, 56f., 59, 75, 91-100, 102f., 113, 153-159

Schneider, Peter 27, 79-82, 84-90

Schröder, Gerhard (Bundesaußenminister) 20

Schröder, Gerhard (Bundeskanzler) 37

Schumann, Maurice 106-108, 126f., 130

Schumann, Robert $\quad 150$

Schwartz, Laurent 145 
Seillière, Ernest Antoine $27 \mathrm{f}$.

Serisé, Jean 27

Servan-Schreiber, Jean-Jacques $\quad 8,30,38$

Smoydzin, Werner 165-167, 169, 171

Sorman, Guy 30

Soutou, Jean-Marie $\quad$ 100-102

Springer, Axel $\quad 79,89$

Stoleru, Lionel 65

Strauß, Franz Josef $\quad 14,19,153$

Sudreau, Pierre 70,72

Suleiman, Ezra 27

Thatcher, Margaret $\quad 26,29$

Timm, Uwe 79, 127, 133
Védrine, Hubert 117

Vetter, Heinz-Oskar 41

Vidal-Naquet, Pierre 145

Vladimir Lenin 157

Vogel, Joachim 39

Wagenbach, Klaus 79

Waldheim, Kurt 127

Wehner, Herbert 5, 33

Weiss, Peter 87

Wresinski, Joseph 66

Zola, Emile 62 


\section{Autorinnen und Autoren}

Bernhard Gotto, Wissenschaftlicher Mitarbeiter am Institut für Zeitgeschichte München - Berlin

Pierre Guillaume, Honorarprofessor für Zeitgeschichte an der Universität Michel de Montaigne Bordeaux 3

Sylvie Guillaume, emeritierte Professorin für Zeitgeschichte an der Universität Michel de Montaigne Bordeaux 3

Veronika Heyde, Lehrbeauftragte an der Ludwig-Maximilians-Universität München

Markus Lammert, Stipendiat des Bundesministeriums für Bildung und Forschung am College of Europe in Natolin/Warschau

Hélène Miard-Delacroix, Professorin an der Universität Paris IV, Sorbonne

Horst Möller, emeritierter Professor für Neuere und Neueste Geschichte an der Ludwigs-Maximilians-Universität München, 1992 bis 2011 Direktor des Instituts für Zeitgeschichte München - Berlin

Jean Mondot, emeritierter Professor für Germanistik an der Universität Michel de Montaigne Bordeaux 3, ehemaliger Präsident der Société Internationale d'études du XVIII siècle

Eva Oberloskamp, Wissenschaftliche Mitarbeiterin am Institut für Zeitgeschichte München Berlin

Nicole Pelletier, Professorin für Germanistik an der Universität Michel de Montaigne Bordeaux 3, Leiterin der Forschungsgruppe CLARE (Cultures Littératures Arts Représentations Esthétiques)

Bernard Poloni, Professor an der Universität Paris IV, Sorbonne

Verena Sattler, Wissenschaftliche Mitarbeiterin am Institut für Zeitgeschichte München - Berlin

Jean-Francois Sirinelli, Professor für Zeitgeschichte am Institut d'études politiques de Paris, Direktor des Centre d'histoire de Sciences Po

Jean-Henri Soutou, emeritierter Professor für Zeitgeschichte an der Universität Paris IV, Sorbonne

Udo Wengst, emeritierter Honorarprofessor für Zeitgeschichte an der Universität Regensburg, 1992 bis 2012 stellvertretender Direktor am Institut für Zeitgeschichte München - Berlin 